\title{
EVERY $L$-SPACE IS ISOMORPHIC TO A STRICTLY CONVEX SPACE
}

\author{
MAHLON M. DAY
}

In the preceding paper ${ }^{1}$ a normed linear space $B$ is called sc (sm) if $B$ is isomorphic to a space for which the unit sphere is strictly convex (smooth). Many special spaces were investigated to determine which, if either, of these properties they possessed, but at that time the behavior of the spaces of summable functions over arbitrary measure spaces was not determined in full.

Let $\mu$ be a measure defined over the class of measurable sets in some space $X$. It was proved in SCM, Theorem 11, that $L(\mu)$, the space of $\mu$-summable functions on $X$, is sm if and only if $X$ is $\sigma$-finite under $\mu$; that is, if and only if $X$ is the union of countably many sets of finite $\mu$-measure. It was also proved in SCM, (v) that every $L(\mu)$ is sc if and only if every $L(\phi)$ is sc, where $\phi$ is a measure for which the measure of the whole space is finite. In this paper we use some results of Maharam ${ }^{2}$ on the structure of measure algebras to prove that every $L(\phi)$ is sc. This gives the necessary information to determine the position of the $L(\mu)$ spaces in the table of the introduction of SCM, and gives the general result stated in the title of this note.

The theorems of Maharam which we need for our proof are stated in terms of measure algebras. Maharam, Theorem 2, asserts that a measure algebra which is nonatomic is a union of countably many homogeneous measure algebras. Maharam, Theorem 1, characterizes homogeneous measures as simple product measures. Since we are interested in the $L$-space over the measure algebra rather than in the measure algebra itself, we shall state here the results we want in terms of $L$-spaces.

If $I$ is an index set, let $2^{I}$ be the product of $I$ copies of the set containing only the real numbers -1 and 1 . In $2^{I}$ define the product measure $\pi$ of $I$ copies of the elementary measure which assigns to each of the points -1 and 1 the measure $1 / 2$. Then if $X$ is a space with finite, nonatomic measure $\phi$, Maharam's results assert that $L(\phi)$ can be written as a countable direct sum $L(\phi)=\sum_{n>0} L_{n}$ so that

Presented to the Society, August 30, 1955; received by the editors June 15, 1955.

1 Strict convexity and smoothness of normed spaces, Trans. Amer. Math. Soc. vol. 78 (1955) pp. 516-528. That paper will be referred to throughout the remainder of this paper as SCM; see it for a list of papers pertinent to this subject.

${ }^{2}$ D. Maharam, On homogeneous measure algebras, Proc. Nat. Acad. Sci. U.S.A. vol. 28 (1942) pp. 108-111. 
(a) if $g \in L(\phi)$ corresponds to $\left\{g_{n}\right\}$, where for each $n g_{n} \in L_{n}$, then $\|g\|=\sum_{n}\left\|g_{n}\right\|$, and (b) for each $n L_{n}$ is a space $L\left(\phi_{n}\right)$ where $\phi_{n}$ is defined on a product space $2^{I_{n}}$ and on that space $\phi_{n}$ is a multiple, $c_{n} \pi_{n}$, of the product measure, where also each $c_{n} \geqq 0$, and for each $m \neq n$ the cardinal number of $I_{m}$ is not that of $I_{n}$.

Hence, if $\phi$ is a finite measure, then $L(\phi)$ is the $l_{1}$-sum $\sum_{n \geqq 0} L_{n}$, where $L_{0}=L\left(\phi_{0}\right)$ and $\phi_{0}$ is the atomic part of $\phi$; where for $n>0$, $L_{n}=L\left(\phi_{n}\right)$ and $\phi_{n}$ is a multiple, $\phi_{n}=c_{n} \pi_{n}$, of product measure on a space $2^{I_{n}}$; where also each $c_{n} \geqq 0$ and where if $m \neq n$ the cardinal number of $I_{m}$ is not that of $I_{n}$. Theorem 4 of SCM asserts that the separable space $L_{0}$ is sc. The Corollary of Theorem 6 of SCM asserts that the $l_{1}$-sum of sc spaces is sc. Since for each $n$ with $c_{n}>0 L\left(\phi_{n}\right)$ is isomorphic to $L\left(\pi_{n}\right)$, each $L\left(\phi_{n}\right)$ is sc if each $L(\pi)$ is sc. It follows from this reduction that to prove every $L(\phi)$ sc it suffices to prove every $L(\pi) \mathrm{sc}$, no matter how large the index set I may be.

Let $J$ consist of all the finite subsets $j$ of $I$, including the empty subset $\varnothing$; define $f \varnothing(x)=1$ for all $x$ in $2^{I}$; for each $i$ in $I$ define $f_{i}(x)$ $=i$ th coordinate of $x$; define $f_{j}(x)=\prod_{i \in j} f_{i}(x)$ for all $x$ in $2^{I}$ if $j$ is a nonempty member of $J$. For each $g$ in $L(\pi)$ let $U g$ be the function on $J$ defined for each $j$ by $U g(j)=\int f_{j} g d \pi$, where the integral extends over all of $2^{I}$.

It will suffice for our purposes to prove that the function $U$ has the following properties:

(1) For each $g$ in $L(\pi) U g$ is in $c_{0}(J)$, the set of functions vanishing at infinity on the discrete space $J$,

(2) For each $g$ in $L(\pi)\|U g\|_{c_{0}(J)} \leqq\|g\|_{L(\pi)}$,

(3) If $g \in L(\pi)$ and $U g=0$, then $\|g\|=0$.

The proof that (1) is true is essentially the proof that the Fourier coefficients of a summable function tend to zero. To give the proof in detail, observe first that for each $j|U g(j)|=\left|\int f_{j} g d \pi\right| \leqq \int|g| d \pi$ $=\|g\|_{L(\pi)}$, because $\left|f_{j}(x)\right|=1$ for all $j$ and all $x$ in $2^{I}$. This proves that $U g \in m(J)$, the space of bounded real functions on $J$, and that $\|U g\|_{m(J)}=\operatorname{lub}_{j \in J}|U g(j)| \leqq\|g\|_{L(\pi)}$. But for arbitrary $j$ and $k$ in $J$ set $m$ equal to the symmetric difference of $j$ and $k$; that is, $i \in m$ if and only if $i$ is in precisely one of the sets $j$ and $k$; then the product $f_{j}(x) f_{k}(x)=f_{m}(x)$ for all $x$ in $2^{I}$. Since the $f_{i}, i \in I$, are independent functions on $2^{I}$,

$$
\int f_{i} f_{k} d \pi=\int f_{m} d \pi=\prod_{i \in m} \int f_{i} d \pi=0 \text { if } j \neq k .
$$

Hence the functions $f_{j}$ and $f_{k}$ are orthogonal elements of $L^{2}(\pi)$ if $j \neq k$. Now if $g \in L^{2}(\pi)$, Bessel's inequality shows that $U g \in l_{2}(J)$; this 
set, in turn, is a subset of $c_{0}(J)$. Then for $g$ in $L(\pi)$, let $g_{n}$ be the function defined by truncating $g$ at $-n$ and at $n$; then each $g_{n} \in L^{2}(\pi)$ and $\left\|g-g_{n}\right\|_{L(\pi)}$ tends to zero. Hence $U g_{n} \in c_{0}(J)$ for each $n$ and

$$
\lim _{n}\left\|U g-U g_{n}\right\|_{m(J)}=0 .
$$

Since $c_{0}(J)$ is closed in $m(J)$, this completes the proof of (1) and (2).

To prove (3) observe that the functions $0, f \varnothing$, and $f \varnothing \pm f_{i}$ are the characteristic functions of the only measurable sets which depend only on the coordinate $i \in I$. Hence for $j$ a finite subset of $J$ the measurable sets which depend only on the coordinates $i$ in $j$ have for characteristic functions polynomials in $f \varnothing$ and $f_{i}, i \in J$; these are linear combinations of the functions $f_{k}, k \subseteq j$. Now take a $g$ in $L(\pi)$ such that $U g=0$. Then for any set $M$ depending on only a finite set $j$ of coordinates there is a linear combination $p$ in the functions $f_{k}$, $k \subseteq j$, such that $\int_{M} g d \pi=\int p g d \pi=0$. But these sets $M$ determine the measurable sets in the sense that every $\pi$-measurable set can be covered arbitrarily closely in measure by an $M_{\sigma}$ set. Because the indefinite integral of $g$ is an absolutely continuous set function with respect to product measure $\pi$, this means that for every measurable set $E \int_{E} g d \pi=0$. Setting $E=\{x \mid g(x)>0\}$,

$$
\|g\|=\int|g| d \pi=\int_{E} g d \pi-\int_{2^{I}-E} g d \pi=0 .
$$

Now the conditions (1) to (3) assert that $U$ is a one-to-one, linear transformation of $L(\pi)$ into $c_{0}(J)$. Theorem 10 of SCM asserts that $c_{0}(J)$ is sc; (3) of SCM then asserts that $L(\pi)$ is sc.

It should be mentioned that this example does not serve to settle by a counter-example the possibility noted in SCM that all sm spaces might be sc. For $I$ large enough it was shown in SCM that $l_{1}(I)$ is sc but neither it nor its conjugate is sm; there is no example known of an sm space $B$ for which either $B$ or $B^{*}$ is not sc.

UNIVERSITY OF ILLINOIS 